Document downloaded from:

http://hdl.handle.net/10251/47485

This paper must be cited as:

Mellado Romero, AM. (2011). Quantitative colorimetric-imaging analysis of nickel in iron meteorites. Talanta. 83:1575-1579. doi:10.1016/j.talanta.2010.11.058.

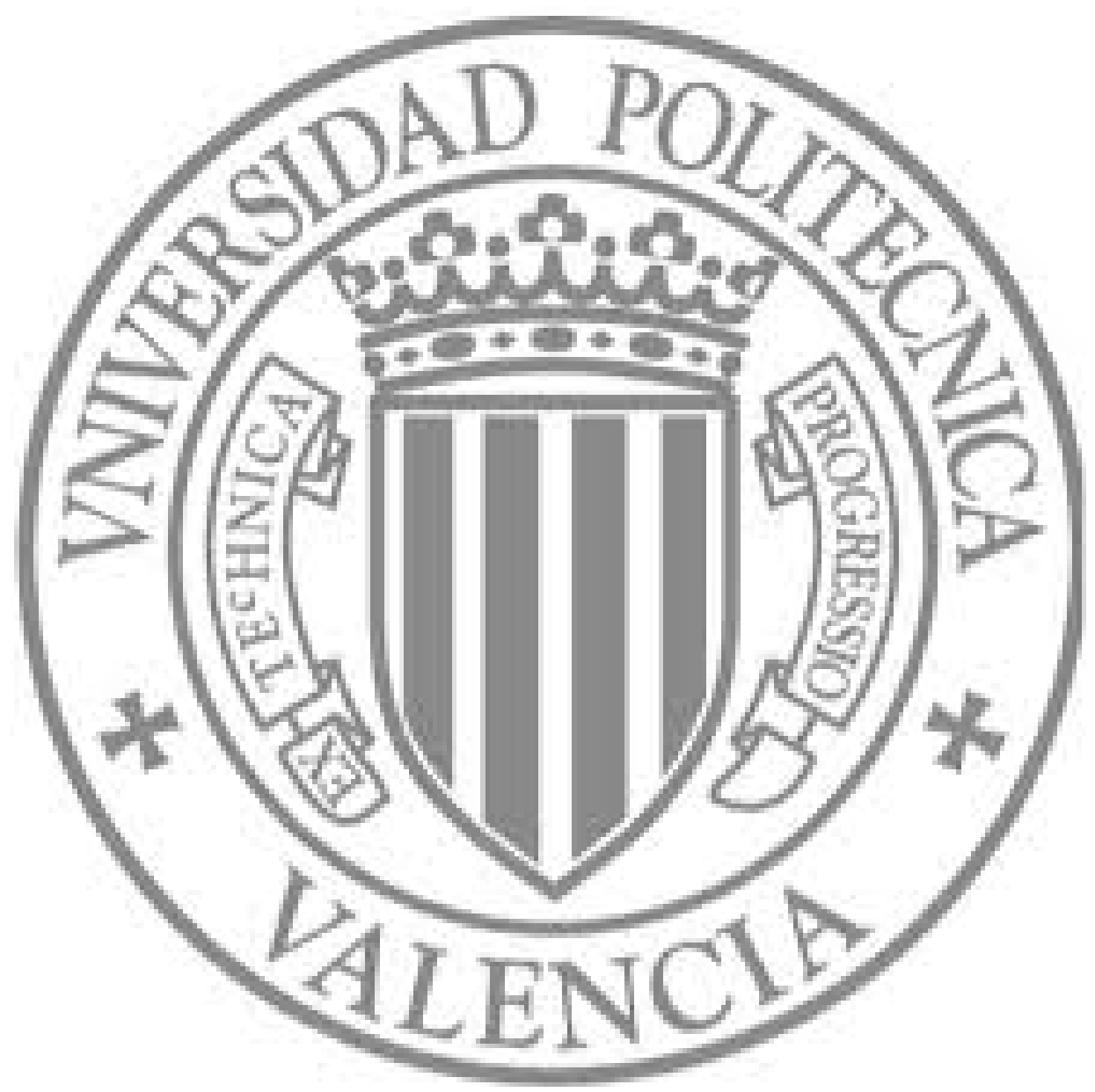

The final publication is available at

http://dx.doi.org/10.1016/j.talanta.2010.11.058

Copyright Elsevier 


\section{Quantitative Colorimetric-Imaging Analysis of Nickel in Iron Meteorites}

Lahuerta Zamora, L., ${ }^{1}$ Alemán López, P., ${ }^{1}$ Antón Fos, G.M., ${ }^{1}$ Martín Algarra, ..${ }^{1}$ Mellado Romero, A.M. ${ }^{2}$ and Martínez Calatayud, $J^{3}$

${ }^{1}$ Departamento de Química, Bioquímica y Biología Molecular. Universidad CEU-Cardenal Herrera. Moncada. Valencia. Spain.

${ }^{2}$ Instituto de Ciencia y Tecnología del Hormigón (ICITECH), Universitat Politècnica de València, València, Spain.

${ }^{3}$ Departamento de Química Analítica, Universidad de Valencia, Valencia, Spain.

\section{Abstract}

A quantitative analytical imaging approach for determining the nickel content of metallic meteorites is proposed. The approach uses a digital image of a series of standard solutions of the nickel-dimethylglyoxime coloured chelate and a meteorite sample solution subjected to the same treatment as the nickel standards for quantitation. The image is processed with suitable software to assign a colour-dependent numerical value (analytical signal) to each standard. Such a value is directly proportional to the analyte concentration, which facilitates construction of a calibration graph where the value for the unknown sample can be interpolated to calculate the nickel content of the meteorite. The results thus obtained were validated by comparison with the official, ISO-endorsed spectrophotometric method for nickel.

The proposed method is fairly simple and inexpensive; in fact, it uses a commercially available digital camera as measuring instrument and the images it provides are processed with highly userfriendly public domain software (specifically, ImageJ, developed by the National Institutes of Health and freely available for download on the Internet).

In a scenario dominated by increasingly sophisticated and expensive equipment, the proposed method provides a cost-effective alternative based on simple, robust hardware that is affordable and can be readily accessed worldwide. This can be especially advantageous for countries were available 
resources for analytical equipment investments are scant. The proposed method is essentially an adaptation of classical chemical analysis to current, straightforward, robust, cost-effective instrumentation.

Keywords: Colorimetric-Imaging analysis, nickel, meteorite, visible spectrophotometry, CCD, ImageJ.

\section{Introduction}

Nickel has been spectrophotometrically determined in a variety of matrices over the last four decades [1, 2]. Dimethylglyoxime (DMG) is one of the most frequently used reagents for this purpose, particularly in the gravimetric determination of the metal, which is a classical method of analysis [3-5]. The reaction of nickel with DMG in an alkaline medium [6-7] has been adapted and widely used for its spectrophotometric determination. The procedure requires the prior oxidation of $\mathrm{Ni}^{2+}$ ion with molecular bromine or iodine (or even persulphate), unless the Ni-DMG complex formed is subjected to liquidliquid extraction with an organic solvent; however, including an extraction step in the process increases analysis times and raises the risk of contamination [8].

The Ni-DMG reaction is the basis for the determination of nickel in steel and cast iron endorsed by the International Standardization Organization (ISO) [9]. The ISO method involves dissolving the solid sample in a mixture of mineral acids (hydrochloric, nitric and perchloric) and the formation of a coloured complex between Ni(III) and DMG in ammonium hydroxide containing molecular iodine and potassium iodide, which is monitored via absorbance readings at $535 \mathrm{~nm}$. The potential interference of cobalt, copper, manganese, chromium, iron and zinc is avoided by adding ammonium citrate to the medium [9, $10]$.

Meteorites are fragments of interplanetary material (which are generically designated "meteoroids") that resist friction heating during their transit of the atmosphere and eventually reach the Earth's surface, leaving a streak of light behind -a phenomenon known as “meteor” [11]. 
Astronomical and laboratory studies have revealed that more than 99\% of all meteorites collected to date are asteroid fragments [12]. Some studies have even allowed the asteroid types they originated from to be identified [13]. Meteorites have traditionally been classified as rocky, metallic or mixed depending on the proportion of characteristic rocks and metals they contain. Each meteorite category in turn encompasses a wide variety of classes and groups established in terms of chemical, mineralogical or structural criteria [14]. Metallic meteorites essentially consist of an Fe-Ni alloy similar to that of the Earth's core, where iron is by far the most abundant element. However, their relative high content in nickel (typically 5-35\%) clearly distinguishes metallic meteorites from man-made Fe-containing materials, which usually contain under 1\% nickel [15].

The metallic meteorite known as "Campo del Cielo", a fragment of which was the subject of the present study, struck the earth in northeastern Argentina $3950 \pm 90$ years ago. Some studies have estimated that the meteorite was about $3 \mathrm{~m}$ in radius and weighed about 883 tons. Various fragments allegedly resulting from its violent interaction with the atmosphere have been found and examined. Many have been acquired by museums or private collectors, and some, small specimens, marketed [16]. To the authors' knowledge, analyses of various samples from the original meteorite have revealed nickel contents ranging from 5.1 to $12.7 \%$ [17-20].

In the present work, we developed a quantitation method which uses imaging analysis to determine nickel with dimethylglyoxime. The method is based on a procedure similar to that developed by the authors to assess coloured solutions with photographic means [43] and was applied to real samples (metallic meteorites) for the first time here. The operational procedure involves taking a digital image for a series of standard solutions of the Ni-DMG complex and one solution of the unknown sample treated in the same manner as the standards. The image is then processed with suitable software in order to assign a numerical value (analytical signal) to each colour spot. Such a value is directly proportional to the analyte concentration in each standard, so it can be used to construct a calibration curve. Interpolating the value for the sample into the calibration curve allows the nickel content of the meteorite to be 
determined. The results thus obtained were validated by comparison with those provided by the reference method: ISO 4939-1984 (E) [9].

For researchers working with limited resources, digital photography and image analysis software may provide a quick and easy method to produce moderate-quality quantitative chemical analysis via colorimetry.

At present, some papers describing analytical applications on imaging methods can be found in chemical literature; some research laboratories have proposed their use in chemiluminescence [21] Raman spectroscopy [22], and electron transmission microscopy [23]. On the other hand and dealing on biological applications, the capturing of digital images of UV fluorescent substances by means of CCDs have been successfully applied to electrophoretic separations of nucleic acids and proteins [24] or to obtain digital images for the quantitation of chemical species following TLC or HPTLC separation [2528]. In this case, CCD cameras have lately been proposed to examine TLC plates in detection modes including transmission, reflectance and fluorescence (under UV light). Digital imaging in the reflectance mode has proved a to be competitive to scanning densitometry [25].

As regards commercial availability, chemical imaging analysis has so far been used with inductively coupled plasma atomic emission spectrometers (ICP-AES) [29], mass spectrometers [30] and high speed screening systems [31]. On the remote sensing field, digital imaging has been applied to evaluation of the photosynthetic biomass [32]; to the quantitative determination of chlorophyll quantitation [33]; and, to the analysis of vegetation [34]. Also, some global imaging systems have allowed the monitoring of water vapor in the atmosphere or deforestation advance by examining the "spectroscopic signature" of the target compounds [35, 36].

The use of digital imaging obtained from cost-effective WebCams, has been used as alternative to evaluate the colour variation during the acidic titrations with no relevant differences with colorimetric monitoring at the 95\% level [37]. Another original alternative to spectrophotometric analysis was the use of a WebCam as a detector and as light source the computer monitor [38]. A consumer digital 
camera and ImageJ, has recently been implemented as a simple, inexpensive alternative to fluorimetry in teaching laboratories [39].

Although the use of consumer digital cameras as quantitative analytical tool has not been widely proposed, they have been yet proposed in different analytical related fields, including forensic science [40, 41] and telemedicine [42].

\section{Experimental}

\subsection{Reagents and equipment}

All reagents used were analytically pure unless stated otherwise. $\mathrm{HCl}, \mathrm{HNO}_{3}, \mathrm{HClO}_{4}, \mathrm{NH}_{3}, \mathrm{I}_{2}, \mathrm{KI}$, $\mathrm{Ni}\left(\mathrm{NO}_{3}\right)_{3}$ and dimethylglyoxime were obtained (from Panreac), and citric acid monohydrate (from Fluka). Solutions were prepared in purified water by reversed osmosis which was de-ionized to 18 $\mathrm{M} \Omega \cdot \mathrm{cm}$ with a Sybron/Barnstead Nanopure II water purification system furnished with a fibre filter of $0.2 \mu \mathrm{m}$ pore size.

The meteorite sample was acquired from the Litos company (Spain, http://www.litos.net, accessed July 2010), which certified it as a fragment of the meteorite "Campo del Cielo" (Chaco Gulamba, Santiago del Estero, Argentina).

Absorbance measurements were made with a Hewlett-Packard 8452A diode array UV-Vis spectrophotometer.

A white porcelain spot plate was used as holder for the colorimetric standards. The plate was $11 \times$ $9 \mathrm{~cm}$ and had twelve $1 \mathrm{~mL}$ wells.

Solutions were transferred with a $1000 \mu \mathrm{L}$ Accumax micropipette.

All digital images were taken with a Nikon Coolpix E995 camera and processed with the public domain software ImageJ, Windows version, developed by the National Institutes of Health and available for free download at http://rsbweb.nih.gov/ij (accessed July 2010). 


\subsection{Procedures}

\subsubsection{ISO reference method}

The standard spectrophotometric method for the quantitation of nickel in steel and cast iron is the classified and described as ISO 4939-1984 (E). The experimental procedure describes the sample solution in a mixture of mineral acids $\left(\mathrm{HCl}, \mathrm{HNO}_{3}\right.$ and $\left.\mathrm{HClO}_{4}\right)$ and then the resulting solution reacts with dimethylglyoxime in an $\mathrm{NH}_{4} \mathrm{OH}$ medium containing $\mathrm{I}_{2}$ and $\mathrm{KI}$. The obtained coloured Ni-DMG chelate was monitored after a reaction time of $10 \mathrm{~min}$. Outputs were obtained at $535 \mathrm{~nm}$ by means of a diode array spectrophotometer. The procedure proposed for nickel standards, which contained the metal at concentrations from 2 to $18 \mathrm{mg} \mathrm{L}^{-1}$, was subjected to the same treatment as the reported for the sample.

\subsubsection{Imaging method}

To check the method validity both absorbance measurements and digital images were obtained for each sample and standard; these images were recorded with a total reaction time under 20 minutes. To this end, the developed coloured solutions were placed in a porcelain spot plate with $1 \mathrm{~mL}$ wells; all plates were white to avoid visual interferences. This operating way facilitated the simultaneous capturing in a single image of all calibration standards and sample solutions under identical lighting conditions. In addition the procedure required only a small volume of solution; a volume of $1 \mathrm{~mL}$ of solution was measured with a micropipette and transferred to one of the plate wells.

The use of flash light would have caused reflections on solution surfaces, due to that, all lighting was provided by fluorescent strips; and to minimize the effect of potential reflections of the strips on the solutions, a diffusing screen was placed over the camera and plate. The screen was a sheet of white paper filter. This setup provided soft lighting; and the use of the largest aperture (i.e. smallest F-number) on the camera lens minimized the exposure times. This performance avoided too dark (underexposed) or too light (overexposed) images according to the recommendations from the camera manufacturers. The camera was placed at $20 \mathrm{~cm}$ from the plate on a static support to ensure reproducible framing and shot under identical conditions: namely, F/3.1 as lens aperture and 1/15 s as exposure time. 
Although the optimum shutter speed suggested by the camera for manual operation was $1 / 15 \mathrm{~s}$, two additional shots were also obtained; one with the stop larger (1/8 s) and another one shorter (1/30 s). This procedure allowed us to examine the potential effect of the exposure time on image quality. Further increasing ( $1 \mathrm{~s})$ or decreasing (1/60 s) on the exposure time led us to obtain useless images which were “burnt out” or too dark, respectively. An aperture of F3.1 was therefore used, in combination with a shutter speed of 1/8 s (Image 1), 1/15 s (Image 2) or 1/30 s (Image 3), to obtain the photographs for the standards and unknown sample following delivery to the plate wells.

Each image thus obtained was processed in triplicate by four different operators (A-D) using the software ImageJ. The procedure was as follows: each operator split each colour image into three channels: red, green and blue (RGB). The image of the green channel -green was the opposite of the solution colour, reddish ( $\lambda=535 \mathrm{~nm}$ )- was colour-reversed with ImageJ to ensure a positive slope in the final calibration graph; then, each operator made neutral grey measurements of a uniform elliptical area (see Fig. 2) for each standard (1-5) and the sample (S) in each plate well.

The calibration curve thus obtained was used to interpolate the measurement for the sample to calculate its nickel concentration. The overall results (12 calibrations per image) are illustrated and discussed in next paragraphs.

\subsection{Data processing}

Groups of results were statistically compared via one-way analysis of variance; when significant differences were found, the Newman-Keuls multiple comparison test was used to identify those significant at the 0.05 level or higher.

\section{Results and discussion}

\subsection{ISO reference method}

The calibration curve provided by the ISO reference method [1] fitted the equation

$$
A=0.0934 C+0.0751\left(\mathrm{C} \text {, means the nickel concentration in } \mathrm{mg} \mathrm{L}^{-1}\right)
$$


with $r=0.998$. Interpolation of the measured absorbance (average of three replications) for the sample

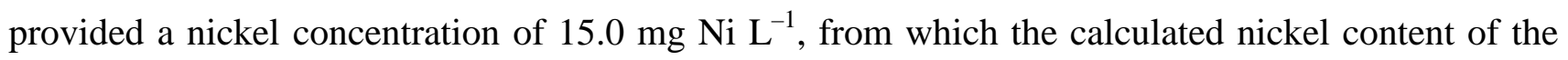
meteorite sample was $7.7 \%$. This result is consistent with formerly reported elsewhere from other fragments [14-17].

\subsection{Imaging method}

By way of example, this section discusses the results obtained by Operator A in one of the three image processing runs on the image taken at a shutter speed of 1/8 s (Image 1). Figure 1 shows the color photograph for the standards (1-5) and sample (S) on the same plate.

In order to develop a simplified procedure, blank solution was not included because of its colour was negligible. On the other hand, we must bear in mind that the subtraction of the blank value from all standards and sample will not affect the sample interpolated concentration.

The operator used the software ImageJ to split the image into its RGB channels and selected the green one for subsequent work. The green channel was then colour-reversed in order to ensure a positive slope in the calibration curve and the neutral grey level was measured over a uniform elliptical area of the spot for each standard and the sample (Fig. 2). The neutral grey levels thus obtained for each standard (15) and the sample (S) are shown in Table 1.

The calibration curve obtained with the proposed method fitted the equation

$$
\mathrm{GL}=8.283 C\left(\mathrm{mg} \mathrm{L}^{-1}\right)+77.428
$$

with $r=0.998$. Interpolation of the grey level (GL) for the sample, 204.713, gave a nickel concentration of $15.4 \mathrm{ppm}$, from which the calculated metal content of the meteorite was 7.9\%. Therefore, the error with respect to the ISO reference value, $7.7 \%$, was only $+2.6 \%$.

The proposed method was validated (accuracy, repeatability) by four different operators (A-D) process three different images to obtain as many calibration curves for each (a total of 12 curves per image). Each curve was used to interpolate the concentration of the meteorite sample to calculate the relative error with respect to the ISO reference method. Table 2 shows the average slope and nickel 
concentration in the sample $\left(C_{s}\right)$ as obtained by the operators from Images 1-3, their corresponding coefficients of variation $(\mathrm{CV})$ and the percent relative errors $\left(E_{\mathrm{r}}\right)$ with respect to the ISO method.

Table 3 shows the average nickel concentration in the sample $\left(C_{\mathrm{s}}\right)$ as obtained by the four operators from different images (1-3), together with the corresponding coefficients of variation (CV) and percent relative errors with respect to the ISO method.

The statistical analysis of the results revealed the presence of significant differences between Image 3 and the other two (Table 2). The differences can be ascribed to the different exposure time used to obtain Image 3, which was shorter than the optimum value suggested by the camera. This reduced the sensitivity of the proposed method (the slope of the calibration curve was $43 \%$ lower for Image 3 than for Image 1) and increased the error with respect to the ISO reference method by $165 \%$ between the two images. There were, however, no significant differences between Images 1 and 2.

As can be seen from Table 3, there were significant differences between the results of Operators B and $\mathrm{D}$, but not between those of $\mathrm{A}$ and $\mathrm{C}$ or these two and the previous ones. The coefficients of variation were all lower than $2.25 \%$ and the relative errors never exceeded $+4 \%$.

Based on the data of Tables 2 and 3, the proposed method is acceptably accurate and repeatable; in fact, CVs never exceeded $2.25 \%$ and errors were all below $+5.3 \%$ with any operator and exposure time. However, the best results were those provided by Image 1, which was taken under conditions of slight overexposure. If only the results for this image are considered (Table 4), then repeatability was good both between calibrations by the same operator (CVs were all less than 1.70\%) and between operators (the average result for the three was $15.32 \pm 0.10$, with $\mathrm{CV}=0.65 \%$ ); also, the relative errors with respect to the ISO reference method were $+2.1 \%$ on average and none exceeded $+2.7 \%$.

\section{Conclusions}

Based on the overall results obtained in this work, the proposed imaging method for the quantitation of nickel in meteorite samples performs on a par with the ISO reference method for nickel. However, the proposed method has the significant advantage of its ease of implementation and low cost; in fact, it 
uses a commercial digital camera and a personal computer as measuring instruments, and both are more inexpensive and widely available than the spectrophotometers usually required to determine nickel. In addition, the photographs taken can be processed with very user-friendly, public domain software (specifically, ImageJ, developed by the National Institutes of Health and freely available on the Internet).

Also, the proposed method is extremely flexible and has an enormous analytical potential. Any classical colorimetric method can be potentially adapted to the imaging methodology employed to determine nickel here in order to quantify a variety of other analytes (e.g. those determined in trace amounts with organic reagents [10] or analysed in a qualitative or semi-quantitative manner in classical spot tests $[44,45])$. The proposed method provides a simple, fairly expeditious, extremely cost-effective tool for their quantitation. The results obtained so far testify to its high potential and have encouraged the authors to undertake the development of a new method to measure light absorption and scattering by precipitates and coloured solids. The preliminary results obtained so far are quite promising. The method described in this paper is a forward step on a former experiment and can be proposed as a new analytical procedure with a wide applicability.

In a scenario involving the use of increasingly sophisticated -and expensive- equipment, the proposed methodology constitutes a "solidary" alternative inasmuch as it affords quantitative determinations in domains such as student laboratories and developing countries, where resources for purchasing and maintaining spectrophotometers are scant and users compelled to use commercial kits (test methods) that are usually only semi-quantitative and relatively expensive.

\section{References}

[1] R. Khanam, R. Singh, A. Mehta, R. Dashora, R. S. Chauhan, A. K. Goswami, Rev. Anal. Chem. 24 (2005) 149.

[2] D. N. Purohit, D. K. Gupta, I.R. Bishnoi, Rev. Anal. Chem. 9 (1989) 189.

[3] L. Tschugaeff, Z. Anorg. Chem. 46 (1905) 144.

[4] O. Z. Brunck, Z. Angew. Chem. 20 (1907) 824. 
[5] J. Hemmingsen, D. Larkin, T. Martin, Anal. Chem. 58 (1986) 2087.

[6] Z. Marczenko, Spectrophotometric Determination of Elements, Wiley, New York (1976) 372.

[7] E. Booth, J. D. H. Strickland, J. Am. Chem. Soc. 75 (1953) 3017.

[8] E. B. Sandell, R. W. Perch, Ind. Eng. Chem., Anal. Ed. 11 (1939) 309.

[9] International Standard (ISO) 4939.Steel and cast iron - Determination of nickel content Dimethylglyoxime spectrophotometric method. Ref. No. ISO 4939 - 1984 (E).

[10] J. Fries, H. Getrost, Organic Reagents for Trace Analysis, E. Merck Darmstadt, 1977.

[11] “Meteorite”. Encyclopaedia Britannica. 2009.

[12] A. Bevan, J. De Laeter, Meteorites: A Journey Through Space and Time. Smithsonian Institution Press / University of New South Wales, Sydney, 2002.

[13] H. McSween, Meteorites and Their Parent Planets, Cambridge University Press, 1999.

[14] R. Hutchison, Meteorites: A Petrologic, Chemical and Isotopic Synthesis, Cambridge University Press, 2007.

[15] F. Heide, F. Wlotzka, Meteorites: messengers from space, Springer, 1995.

[16] E. D. Cabanillas, T. A. Palacios, Planet. Space Sci., 54 (2006) 303.

[17] E. H. Ducloux, Rev. Fac. Cienc. Quim. Univ. Nac. La Plata, 5 (1928) 8.

[18] J. T. Wasson, Icarus, 12 (1970) 407.

[19] B. G. Choi, X. Ouyang, J. T. Wasson, Geochim. Cosmochim. Acta, 59 (1995) 593.

[20] J. T. Wasson, G. W. Kallemeyn, Geochim. Cosmochim. Acta, 66 (2002) 2445.

[21] T. Goldmann, A. Zyzik, S. Loeschke, W. Lindsay, E. Vollmer, J. Biochem. Biophys. Meth. 50 (2001)91.

[22] S. Schlücker, M. D. Schaeberle, S. W. Huffman, I. W. Levin, Anal. Chem.75 (2003) 4312.

[23] G. Y. Fan, M. H. Ellisman, J. Microsc. 200 (2000) 1.

[24] B. Chakravarti, M. Loie, W. Ratanaprayul, A. Raval, S. Gallagher, D. N. Chakravarti, Proteomics 8 (2008) 1789.

[25] M. Lancaster, D. M. Goodall, E. T. Bergström, S. McCrossen, P. Myers, Anal. Chem. 78 (2006) 905. 
[26] T. Hayakawa, M. Hirai, Anal. Chem. 75 (2003) 6728.

[27] M. Prosek, I. Vovk, J. Chromatogr. A 768 (1997) 329.

[28] Hess, A.V.I., Journal of Chemical Education, 2007, 84, 842.

[29] Q. S. Hanley, C. W. Earle, F. M. Pennebaker, S. P. Madden, M. B. Denton, Anal. Chem. 68 (1966) A661.

[30] D. W. Koppenaal, C. J. Barinaga, M. B. Denton, R. P. Sperline, G. M. Hieftje, G. D. Schilling, F. J. Andrade, J. H. Barnes, Anal. Chem.77 (2005)418A.

[31] S. Merk, A. Lietz, M. Kroner, M. Valler, R. Heilker, Comb. Chem. High Throughput Screening 7 (2004)45.

[32] R. J. Murphy, A. J. Underwood, M. H. Pinkerton, Marine Ecology Progress Series, 2006, 312, 45.

[33] Murphy, R.J., Underwood, A.J., Jackson, A.C., J. Exp. Mar. Biol. Ecol., 2009, 380, 68.

[34] C. Dean, T. A. Warner, J. B. McGraw, ISPRS Journal of photogrammetry and remote sensing, 2000, 55, 105.

[35] Gulf of Maine Ocean Observing System. The Gulf of Maine: As Seen from Space. http://www.gomoos.org/buoy/satellite.html (accessed July 2010).

[36] European Organization for the Exploitation of Meteorological Satellites. EUMETSAT - Access to Data - SEVIRI. http://www.eumetsat.int (accessed July 2010).

[37] E. N. Gaiao, V. L. Martins, W.S. Lyra, L. F. Almeida, E. C. Silva, and M. C. U. Araujo, Analytica Chimica Acta, 2006, 570, 283.

[38] A. Alimelli, D. Filippini, R. Paolesse, S. Moretti, G. Ciolfi, A, D’Amico, I. Lundstrom, C. Di Natale. 2007, Analytica Chimica Acta, 597: 103.

[39] T. Cumberbatch, Q. S. Hanley, J.Chem.Ed.84 (2007) 1319.

[40] T. Yamamoto, H. Takiwaki, S. Arase, and H. Ohshima, Skin Research and Technology, 2008, 14, 26.

[41] J. W. Wagner, G. M. Miskelly. , Journal of Forensic Sciences, 2003, 48, 593.

[42] A. W. Martinez, S. T. Phillips, E. Carrilho, S. W. Thomas, H. Sindi, G. M. Whitesides, Analytical 
Chemistry, 2008, 80, 3699.

[43] L. Lahuerta Zamora, A. M. Mellado Romero, J. Martínez Calatayud, Analytical Letters, In press.

[44] F. Feigl, V. Anger, Spot Tests in Inorganic Analysis. Elsevier. Amsterdam. 1972.

[45] F. Feigl, Spot Tests in Organic Analysis. Elsevier. Amsterdam. 1966. 
Table 1. Neutral grey measurements of the standard solutions (1-5) and sample (S) obtained by Operator A in one of the three processing runs of Image 1.

\begin{tabular}{|c|c|c|}
\hline Solution & $\boldsymbol{C}_{\mathbf{N i}}\left(\mathbf{m g ~ L}^{-\mathbf{1}}\right)$ & Grey level (NG) \\
\hline 1 & 2.0 & 92.091 \\
\hline 2 & 6.0 & 128.700 \\
\hline 3 & 10.0 & 159.476 \\
\hline 4 & 14.0 & 197.850 \\
\hline 5 & 18.0 & 223.177 \\
\hline S (sample) & $C_{\mathrm{s}}$ & 204.713 \\
\hline
\end{tabular}


Table 2. Average slope and nickel concentration in the sample $\left(C_{\mathrm{s}}\right)$ as determined from Images $1-$ 3 , coefficients of variation (CV) and percent relative errors $\left(E_{\mathrm{r}}\right)$ with respect to the ISO reference method, as obtained by Operators A-D using the proposed method.

\begin{tabular}{|l|c|c|c|c|}
\hline Parameter & $\begin{array}{c}\text { Image 1 } \\
\text { (Average A- } \\
\text { D) }\end{array}$ & $\begin{array}{c}\text { Image 2 } \\
\text { (Average A- } \\
\text { D) }\end{array}$ & $\begin{array}{c}\text { Image 3 } \\
\text { (Average A- } \\
\text { D) }\end{array}$ & $\begin{array}{c}\text { Average } \\
\text { Images 1-3 }\end{array}$ \\
\hline Slope & 8.254 & 6.947 & 4.720 & \\
\hline CV slope (\%) & 0.73 & 1.52 & 0.88 & \\
\hline$C_{\mathrm{s}}\left(\mathrm{mg} \mathrm{L}^{-1}\right)$ & $15.3^{\mathrm{a}}$ & $15.4^{\mathrm{a}}$ & $15.8^{\mathrm{b}}$ & 15.5 \\
\hline $\mathrm{CV} \mathrm{C}_{\mathrm{s}}$ & 0.95 & 0.92 & 1.89 & 1.70 \\
\hline$E_{\mathrm{r}}(\%)$ & +2.0 & +2.7 & +5.3 & +3.3 \\
\hline
\end{tabular}

${ }^{\mathrm{a}, \mathrm{b}}$ Groups with different subscripts were statistically different in the parameter concerned at the $5 \%$ significance level 
Table 3. Average nickel concentration in the sample $\left(C_{s}\right)$ as determined by Operators $\mathrm{A}-\mathrm{D}$, coefficients of variation $(\mathrm{CV})$ and percent relative errors $\left(E_{\mathrm{r}}\right)$ with respect to the ISO reference method as obtained by applying the proposed method to Images 1-3.

\begin{tabular}{|l|c|c|c|c|c|}
\hline \multirow{2}{*}{ Parameter } & $\begin{array}{c}\text { Operator } \\
\mathbf{A}\end{array}$ & $\begin{array}{c}\text { Operator } \\
\text { B }\end{array}$ & $\begin{array}{c}\text { Operator } \\
\mathbf{C}\end{array}$ & $\begin{array}{c}\text { Operator } \\
\mathbf{D}\end{array}$ & Group \\
& $\begin{array}{c}\text { Images } \\
\text { (Average } \\
\text { Images } \\
\text { (Average }\end{array}$ & $\begin{array}{c}\text { Images } \\
\text { (Average } \\
\text { Images } \\
\text { average }\end{array}$ & \\
\hline $\mathrm{C}_{\mathrm{s}}\left(\mathrm{mg} \mathrm{L}^{-1}\right)$ & 15.6 & 15.6 & 15.6 & 15.2 & 15.5 \\
\hline $\mathrm{CV} \mathrm{C}_{\mathrm{s}}$ & $2.25^{\text {a.b.c }}$ & $2.14^{\mathrm{b}}$ & $2.13^{\text {a.b.c }}$ & $0.51^{\mathrm{c}}$ & 1.95 \\
\hline$E_{\mathrm{r}}(\%)$ & +4.0 & +4.0 & +4.0 & +1.3 & +3.3 \\
\hline
\end{tabular}

${ }^{\text {a,b,c }}$ Groups with different subscripts were statistically different in the parameter concerned at the $5 \%$ significance level 
Table 4. Average nickel concentration in the sample $\left(C_{\mathrm{s}}\right)$ as determined by Operators $\mathrm{A}-\mathrm{D}$, coefficients of variation $(\mathrm{CV})$ and percent relative errors $\left(E_{\mathrm{r}}\right)$ with respect to the ISO reference method as obtained by applying the proposed method to Image 1 in three separate calibrations per operator.

\begin{tabular}{|l|c|c|c|c|c|}
\hline Parameter & $\begin{array}{c}\text { Operator A } \\
\text { (Image 1) }\end{array}$ & $\begin{array}{c}\text { Operator B } \\
\text { (Image 1) }\end{array}$ & $\begin{array}{c}\text { Operator C } \\
\text { (Image 1) }\end{array}$ & $\begin{array}{c}\text { Operator D } \\
\text { (Image 1) }\end{array}$ & $\begin{array}{c}\text { Group } \\
\text { average }\end{array}$ \\
\hline$C_{\mathrm{s}}\left(\mathrm{mg} \mathrm{L}^{-1}\right)$ & $15.31^{\mathrm{a}}$ & $15.38^{\mathrm{a}}$ & $15.36^{\mathrm{a}}$ & $15.17^{\mathrm{a}}$ & 15.32 \\
\hline $\mathrm{CV}_{\mathrm{s}}$ & 0.39 & 0.26 & 0.46 & 1.70 & 0.65 \\
\hline$E_{\mathrm{r}}(\%)$ & +2.0 & +2.7 & +2.7 & +1.3 & +2.1 \\
\hline
\end{tabular}

a,b,c Groups with different subscripts were statistically different in the parameter concerned at the $5 \%$ significance level 


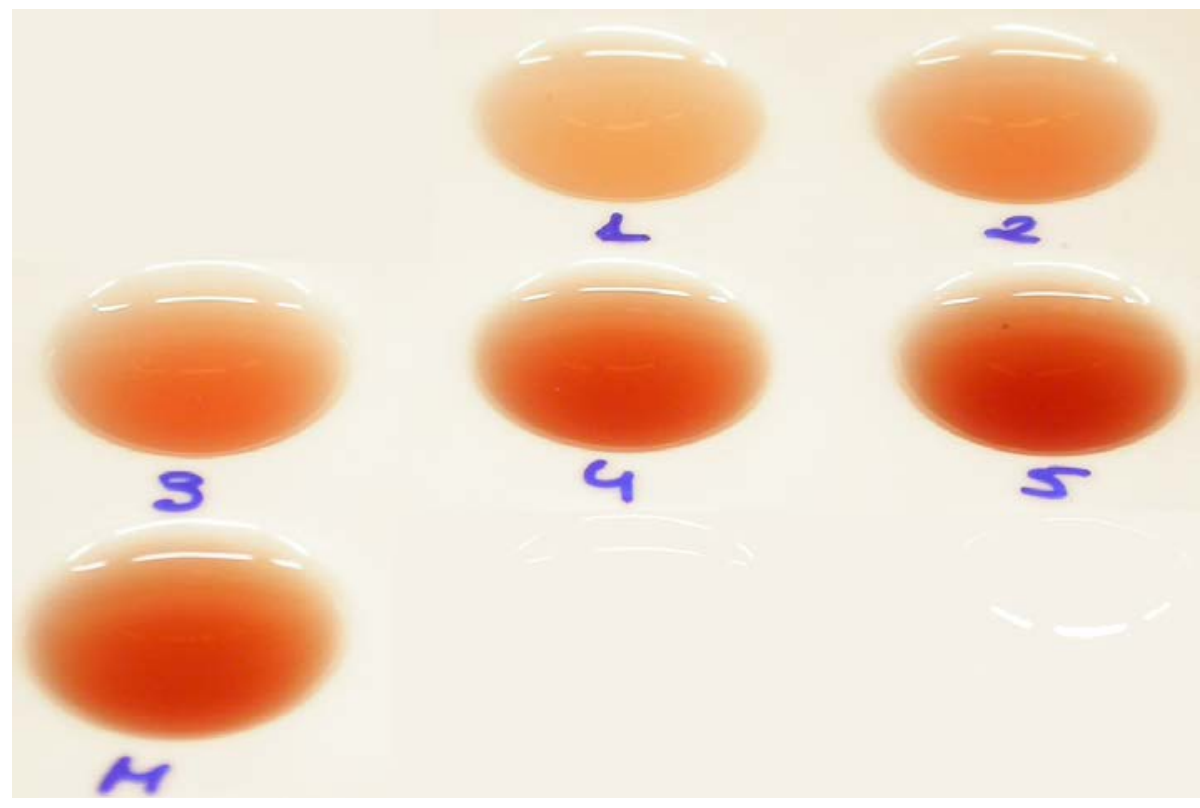

Figure 1. Raw (unprocessed) colour image for the calibration standards (1-5) and sample (M) on the spot plate.

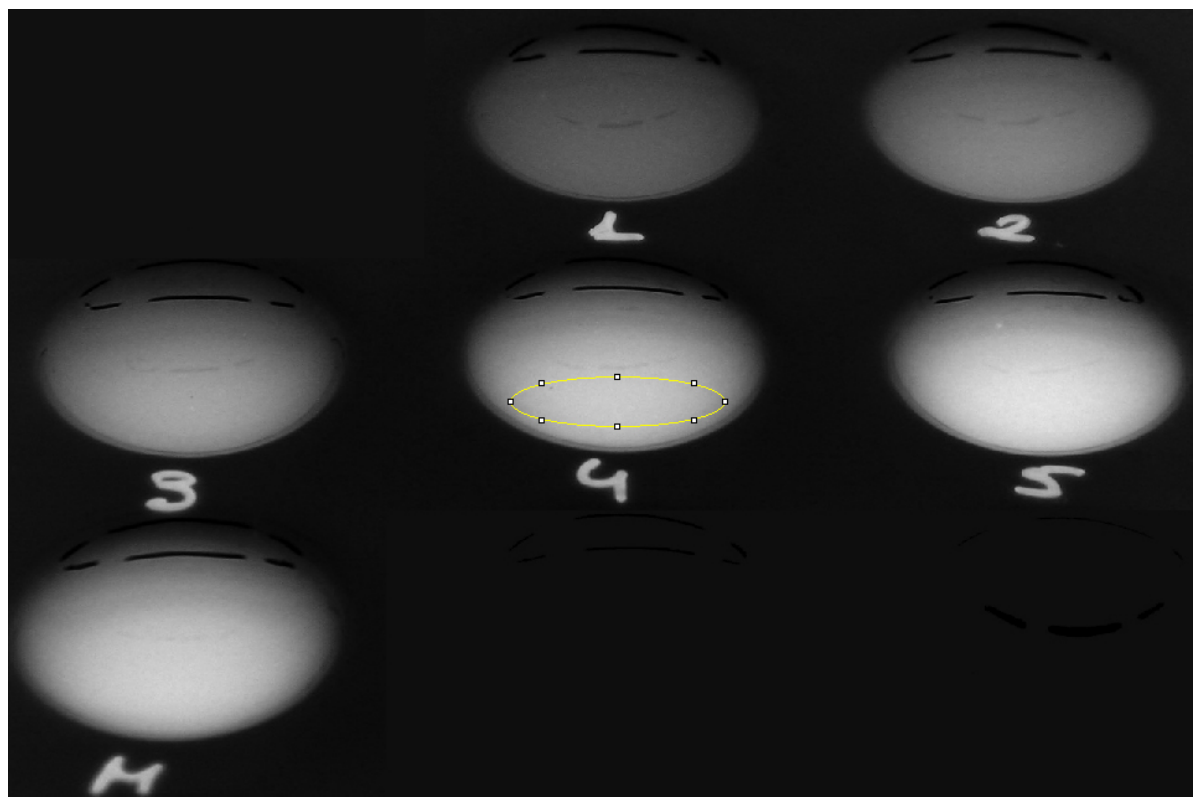

Figure 2. Processed image for the calibration standards (1-5) and sample (M) on the spot plate. Point 4 shows the elliptical area used to measure the neutral grey level for each solution. 